\title{
RF-resonance beam polarimeter Part I. Fundamental concepts
}

\author{
Ya.S. Derbenev \\ Randall Laboratory of Physics, Unicersity of Michigan, Ann Arbor, MI 48109-1120, USA
}

Recelved 23 March 1993

The possibility of an RF-resonance polarimeter (RFP) for fast non-destructuve measurement of beam polarization in an accelerator ring is considered. In order to accumulate the transition radiation from the free oscillating coherent spin of the beam, a passive superconductıng cavity is proposed. The increase of effective voltage in the cavity with tıme (related to beam polarization) is calculated here. The efficiency of the polarimeter does not decrease with beam energy and is proportional to the average beam current A possible scheme of measurement of the accumulated voltage is presented. The noise limitations are taken into account and evaluated. Siberian snakes can be used in order to provıde a sufficiently small value for the spin tune spread. Numerical examples are given

\section{The spin-resonator interaction}

Suppose that a superconducting cavity or a chain of them is introduced into a ring with a cycling bunched charged particle beam. We can describe the dynamic system of particle spins $S^{\jmath}$ and a resonant clectromagnetic mode of the cavity by the Hamiltonian as follows:

$H=\frac{1}{2}\left(P^{2}+\omega_{\mathrm{c}}^{2} Q^{2}\right)+\sum_{J} \boldsymbol{W}_{\mathrm{ext}}^{J} \cdot \boldsymbol{S}^{\jmath}+\sum_{j} \boldsymbol{W}_{\mathrm{c}}^{J} \cdot \boldsymbol{S}^{\jmath}$,

where $P(t)$ and $Q(t)$ are the dynamic canonical variables expressed as functions of time in the presentation of the electromagnetic field $\boldsymbol{E}, \boldsymbol{B}$ of the mode:

$E(r, t)=-\frac{1}{c} P(t) E^{c}(r)$,

$\boldsymbol{B}(\boldsymbol{r}, t)=Q(t) \boldsymbol{\nabla} \times \boldsymbol{E}^{\mathrm{c}} \equiv Q(t) \boldsymbol{B}^{\mathrm{c}}(\boldsymbol{r})$,

$c^{2} \Delta E^{\mathrm{c}}+\omega_{\mathrm{c}}^{2} E^{\mathrm{c}} \equiv 0, \quad \operatorname{div} E^{\mathrm{c}}=0$,

$\int\left(E^{c}\right)^{2} \mathrm{~d}^{3} r=4 \pi, \quad c^{2} \int\left(B^{c}\right)^{2} \mathrm{~d}^{3} r=4 \pi \omega_{\mathrm{c}}^{2}$,

where $\boldsymbol{E}^{\mathfrak{c}}, \boldsymbol{B}^{\mathrm{c}}$ are the eigen-functions and $\omega_{\mathrm{c}}$ is the eigen-frequency of the resonant mode. The Poisson bracket of $P$ and $Q$ is

$\{P, Q\}=1$.

The $\boldsymbol{W}$-vectors describe the spin precession in the electromagnetic field [1]:

$\dot{S}=\{H, S\}=W \times S$, where

$$
\begin{aligned}
\boldsymbol{W}= & -\frac{e}{m c}\left\{\left(G+\frac{1}{\gamma}\right) \boldsymbol{B}_{\perp}+\frac{1+G}{\gamma} \boldsymbol{B}_{\|}\right. \\
& \left.+\left(G+\frac{1}{\gamma+1}\right) \boldsymbol{E} \times \frac{\boldsymbol{v}}{c}\right\},
\end{aligned}
$$

where $e$ and $m$ are particle's charge and mass, $c$ is light velocity, $\gamma^{-1}=\left(1-v^{2} / c^{2}\right)^{1 / 2}, G$ is the anomalous magnetic factor in the relation between particle spin and its magnetic moment:

$\mu=(1+G) \frac{e}{m c} S$,

and $\boldsymbol{B}_{\perp}$ and $\boldsymbol{B}_{\|}$are the transverse and longitudinal components of $\boldsymbol{B}$ with respect to the particle velocity $v$. Note that $G=1.79$ for protons and antiprotons, and $1.13 \times 10^{-3}$ for electrons and positrons.

The vectors $\boldsymbol{W}_{\text {ext }}$ and $\boldsymbol{W}_{\mathrm{c}}$ correspond to the accelerator guide field and cavity field, respectively; the symbol $J$ is for a single particle, while the sum is for all the particles of the beam.

For this paper, we neglect the effects of charge interaction here; they will be studied in Part II. The field equations are then obtained as follows:

$\dot{P}=\{H, P\}=-\omega_{c}^{2} Q-\sum_{\jmath} S^{\jmath} \cdot \frac{\partial \boldsymbol{W}_{\mathrm{c}}^{J}}{\partial Q}$,

$\dot{Q}=\{H, Q\}=P+\sum_{j} S^{\prime} \cdot \frac{\partial W_{\mathrm{c}}}{\partial P}$. 
We assume the spin motion to be stable in the machine field. Then this motion is characterized by free precession with a spin tune $\nu$ around a periodic axis $n(\theta)$ :

$\boldsymbol{S}=S_{n} \boldsymbol{n}(\theta)+\frac{1}{2}\left(S_{+} \boldsymbol{\eta}+S_{-} \boldsymbol{\eta}^{*}\right)$,

where $\boldsymbol{n}(\theta), \boldsymbol{\eta}(\theta)$ and $\boldsymbol{\eta}^{*}(\theta)$ are the vector solutions of the spin equations on a particle closed orbit, with the properties [2]:

$\boldsymbol{n} \cdot \boldsymbol{\eta}=0, \quad \boldsymbol{\eta}^{2}=0, \quad \boldsymbol{\eta} \cdot \boldsymbol{\eta}^{*}=2, \quad \boldsymbol{n}^{2}=1$,

$\boldsymbol{n}(\boldsymbol{\theta})=\boldsymbol{n}(\theta+2 \pi)$

$\boldsymbol{\eta}(\theta+2 \pi)=\boldsymbol{\eta}(\theta) \mathrm{e}^{-2 \pi \nu_{0}}, \quad \boldsymbol{\eta}(\theta)=\boldsymbol{e}(\theta) \mathrm{e}^{-i \nu_{0} \theta}$,

$e(\theta+2 \pi)=e(\theta)$,

where $\theta$ is the generalized azimuth angle along the closed orbit and $\nu_{0}$ is spin tune of the closed orbit. Taking into account the particle free oscillations, the variables $S_{n}, S_{ \pm}$as well as spin tune $\nu$ are not constant in time and are not the same for different particles. We neglected here the spin-orbit coupling effect on $S_{n}$ and $S_{ \pm}$, but have taken into account the spin tune spread $\Delta \nu$. This parameter is important when we study the coherent spin interaction.

We consider now a resonance situation:

$\omega_{\mathrm{c}} \approx \nu_{0} \omega_{0}+k \omega_{0}$,

where $\omega_{0}$ is the frequency of the guiding (or bunching) machine RF-field, and $k$ is an integer. It is then convenient to describe the spin precession with respect to the frame:

$\boldsymbol{n}, \hat{n}_{1}, \hat{\boldsymbol{n}}_{2}$,

where

$\hat{\boldsymbol{n}}_{1}+\mathrm{i} \hat{\boldsymbol{n}}_{2}=\boldsymbol{e} \mathrm{e}^{\mathrm{1}\left(k-\omega_{\mathrm{c}} / \omega_{0}\right) \theta} \equiv \hat{\boldsymbol{n}}, \quad \boldsymbol{S}=S_{n} \boldsymbol{n}+\frac{1}{2}\left(\hat{\boldsymbol{S}} \hat{\boldsymbol{n}}+\hat{S}^{*} \hat{\boldsymbol{n}}^{*}\right)$,

and then the Poisson brackets will be:

$\left\{S_{n}, \hat{S}\right\}=\mathrm{i} \hat{S}, \quad\left\{\hat{S}, \hat{S}^{*}\right\}=2 \mathrm{i} S_{n}$.

We also introduce the complex field amplitudes as follows:

$a=\frac{1}{\sqrt{2 \omega_{\mathrm{c}}}}\left(\mathrm{i} P+\omega_{\mathrm{c}} Q\right) \mathrm{e}^{\omega_{\mathrm{c}} t}, \quad\left\{a, a^{*}\right\}=1$.

Suppose for simplicity's sake, that the resonance electric field is equal to zero near the beam (due to symmetry). Then, we get the reduced Hamiltonian and the equations of motion as follows:

$H_{\mathrm{res}}=\sum_{\jmath} \epsilon, S_{n}^{\prime}+\left[g_{k} a^{*} \sum_{\jmath} \hat{S}^{\prime}+\right.$ c.c. $]$

where

$g_{k} \equiv \frac{e}{2 m c \sqrt{2 \omega_{\mathrm{c}}}}\left\langle\left[\left(G+\frac{1}{\gamma}\right) \boldsymbol{B}_{\perp}^{\mathrm{c}}+\frac{1+G}{\gamma} \boldsymbol{B}_{\|}^{c}\right] \boldsymbol{e} \mathrm{e}^{i k \theta}\right\rangle$,
$\dot{a}=\{H, a\}=-\mathrm{i} g_{k} \sum \hat{S}_{\jmath}$,

$\hat{S}^{\prime}=\mathrm{i} \epsilon_{j} \hat{S}^{\jmath}+2 \mathrm{i} g_{k}^{*} S_{n}^{\prime} a$,

$\dot{S}_{n}^{\prime}=-\mathrm{i} \hat{S}^{\prime} g_{k} a^{*}+$ c.c.,

where the brackets \langle\rangle mean averaging along the beam orbit and the detune is

$\epsilon_{J} \equiv\left(\nu_{\jmath}-\frac{\omega_{c}}{\omega_{0}}+k\right) \omega_{0}$.

One can see that the system under consideration has the following general invariants:

the Hamiltonian $H_{\text {res }}$ itself,

the total beam spin value at $\Delta \nu_{j}=0$,

and the combined adiabatic invariant

$I_{+}=|a|^{2}+\sum S_{n}^{j}$.

\section{RF-voltage accumulation rate}

For further calculations, suppose that we could neglect the $\varepsilon$, spread for a required period of time. The initial conditions are:

$a(0)=0 ; \quad \sum_{j} S_{n}^{\prime}=N \frac{\hbar}{2} \xi \cos \alpha$,

$\sum_{j} \hat{S}^{\jmath}=N \frac{\hbar}{2} \xi \sin \alpha \mathrm{e}^{1 \varphi}$,

where $N$ is the total number of particles, $\xi$ is the degree of beam polarization, $\alpha$ and $\varphi$ are polar and azimuthal angles of coherent spin declination from the periodic axis $n$. One can find, from the eq. (5), that the electromagnetic energy of the cavity $\mathscr{E}_{c}$ will grow with time according to the equation:

$\mathscr{E}_{\mathrm{c}}=\omega_{\mathrm{c}}|a|^{2}=\omega_{\mathrm{c}} N^{2}\left|g_{k}\right|^{2} \frac{\hbar^{2} t^{2}}{4} \xi^{2} \sin ^{2} \alpha$.

To make further headway, we must make certain assumptions on the RF-field geometry.

As one can see from definition (2), the use of $B_{\perp}^{c}$ is effective for all energies of the particle, while the use of $B_{\|}^{c}$ is effective only for $\gamma G \leq 1$ (non-relativistic protons and electrons with $\gamma \leq 10^{3}$ ). Let us now consider the case when $B^{c}$ is transverse. Assume a resonance condition (3) with $\mathrm{TM}_{110}$ mode of a cylindrical resonator [3] with its symmetry axis parallel to the beam direction (see fig. 1). Then we find

$\left|g_{k}\right|=\frac{1}{041}\left(G+\frac{1}{\gamma}\right) \frac{e}{m c} \sqrt{\frac{2 \pi \omega_{\mathrm{c}}}{d}} \frac{1}{L}\left|\sin \frac{\omega_{\mathrm{c}} d}{2 c \beta}\right|$,

where $L$ is the machine circumference and $\mathrm{d}$ is an effective length of the resonator. Now we get from eqs. (8) and (9):

$\mathscr{E}_{\mathrm{c}}(t)=\frac{\pi}{2 d}\left[J\left(G+\frac{1}{\gamma}\right) \frac{\lambda \omega_{\mathrm{c}} t}{0.41 \mathrm{c}} \sin \left(\frac{\omega_{\mathrm{c}} d}{2 \beta c}\right) \xi \sin \alpha\right]^{2}$, 

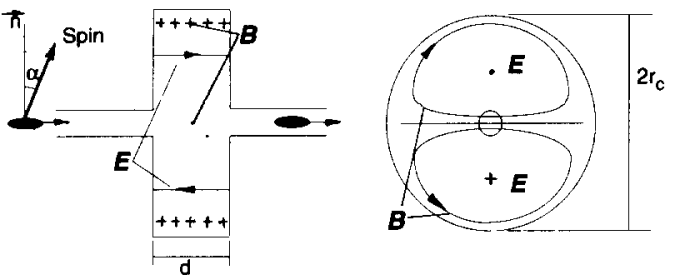

Fig. 1 Scheme of spin interaction with $\mathrm{TM}_{110}$ mode.

where $\beta=v / c, \lambda=\hbar / m c$, and $J$ is the average beam current.

The electromagnetic energy of the resonator is related to the effective voltage $\mathrm{V}$ in the cavity as:

$\frac{1}{2} C V^{2}=\mathscr{E}_{\mathrm{c}}, \quad C=r_{\mathrm{c}}^{2} / 4 d$,

where $C$ is the capacity of the resonator and $r_{c}$ is the cavity's radius. Note that the ratio $\omega_{\mathrm{c}} r_{\mathrm{c}} / c$ for the considered mode is equal to $\approx 3.8$ [3]. Then,

$\dot{V}=33\left(G+\frac{1}{\gamma}\right) \frac{J \lambda}{r_{\mathrm{c}}^{2}} \sin \left(\frac{1.9 d}{\beta r_{\mathrm{c}}}\right) \xi \sin \alpha$.

If the total number of the identical resonators (coupled or non-coupled) is $\mathscr{N}$, the maximum total effective voltage would be $\mathscr{r}$ times larger.

Note that the minimum $r_{\mathrm{c}}$ is limited by the chamber aperture. Also, the bunch length must be less than $r_{\mathrm{c}}$ : $l_{\mathrm{h}} \ll r_{\mathrm{c}}$.

The accumulation rate of the voltage of the cavity considered does not decrease with energy and machine circumference when $\gamma G>1$ and is proportional to the beam current.

\section{Maximum accumulated voltage}

The maximum voltage that can be accumulated in the resonator is defined by an effective maximum time:

$V_{\max }=\dot{V}_{t_{\max }}$,

$t_{\mathrm{max}}=\min \left(\tau_{\mathrm{sp}}, \tau_{\mathrm{c}}\right)$,

where $\tau_{\mathrm{sp}}=\left(\omega_{0} \Delta \nu\right)^{-1}$, and $\tau_{\mathrm{c}}$ is the RF-voltage damping time due to the cavity wall resistance. It is related to the quality factor $Q_{\mathrm{c}}$ of the considered cavity mode as:

$\tau_{\mathrm{c}}=Q_{\mathrm{c}} / \omega_{\mathrm{c}}$.

A typical $Q_{\mathrm{c}}$ value for $\mathrm{TM}_{110}$ mode of available superconducting resonators is $2 \times 10^{10}$ [4]. At $r_{\mathrm{c}}=20$ $\mathrm{cm}, \tau_{\mathrm{c}}$ value is about $5 \mathrm{~s}$.

To provide $\tau_{\mathrm{sp}}$ value compatible with $\tau_{\mathrm{c}}$, special measures for reduction of $\Delta v$ are required. For a bunched beam, $\Delta \nu$ is proportional to the beam emittances. It can be reduced by application of compensating sextupoles. The spin tune spread can be made especially small in rings with Siberian snakes. These possibilities should be investigated in detail separately.

\section{RF-voltage measurement (noise criteria)}

In view of the rather small value of possible accumulated voltage, one has to take into account noise limitations. There are two basic kinds of noise: thermal cavity noise and voltmeter input thermal noise.

1) The cavity noise effect is defined by the noise spectral density of the considered field mode, which is a single oscillator. When there is a state of thermodynamic equilibrium in the cavity walls, we can use a canonical formula for the oscillator energy distribution [5], which can be written for frequencies near resonance as follows:

$\frac{\mathrm{d} \mathscr{E}_{\mathrm{r}}}{\mathrm{d} \omega} \approx \frac{T_{\mathrm{c}} \tau_{\mathrm{c}}}{1+\left(\omega-\omega_{\mathrm{c}}\right)^{2} \tau_{\mathrm{c}}^{2}} \frac{1}{\pi}$,

where $T_{\mathrm{c}}$ is the cavity wall temperature (in units of energy). A voltmeter will integrate the frequencies in the interval $\Delta \omega \sim 1 / t_{\mathrm{m}}$, where $t_{\mathrm{m}}$ is the measurement time:

$t_{\mathrm{m}}=\max \left\{\tau_{\mathrm{sp}}, \tau_{\mathrm{c}}\right\}$.

There are two characteristic cases:

a) $\tau_{\mathrm{sp}} \ll \tau_{\mathrm{c}}$; then

$\left(\mathscr{E}_{\mathrm{c}}\right)_{\mathrm{max}}=\frac{1}{2} C \dot{V}^{2} \tau_{\mathrm{sp}}^{2}, \quad t_{\mathrm{m}}=\tau_{\mathrm{c}}, \quad \mathscr{E}_{\mathrm{T}} \sim T_{\mathrm{c}}$,

b) $\tau_{\mathrm{sp}} \gg \tau_{\mathrm{c}}$; then

$\left(\mathscr{E}_{\mathrm{c}}\right)_{\max }=\frac{1}{2} C \dot{V}^{2} \tau_{\mathrm{c}}^{2}, \quad t_{\mathrm{m}}=\tau_{\mathrm{sp}}, \quad \mathscr{E}_{\mathrm{T}} \sim T_{\mathrm{c}}\left(\tau_{\mathrm{c}} / \tau_{\mathrm{sp}}\right)$.

To measure the accumulated voltage with confidence, the following condition is necessary:

$C \dot{V}^{2} \tau_{\mathrm{sp}} \min \left(\tau_{\mathrm{sp}}, \tau_{\mathrm{c}}\right) \gg T_{\mathrm{c}} / \mathscr{N}$.

2) The frequency bandwidth $\Delta f$ of an applied RFvoltmeter should satisfy the requirement

$2 \pi t_{\mathrm{m}} \Delta f \gg 1$

on the other side, the $\Delta f$ value should be small enough to eliminate the noise voltage due to the input resistance $R_{\mathrm{m}}$ of the voltmeter:

$V_{\mathrm{in}}=\left[2(T R)_{\mathrm{n}} \Delta f\right]^{1 / 2} \ll V_{\max }$.

A possible voltmeter scheme is shown in fig. 2 [6]. In the frequency region $f \sim 10^{8}-10^{9} \mathrm{~Hz}$, the character-

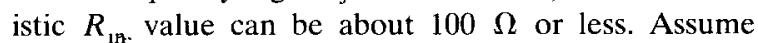
that the preamplifier channel temperature $T_{\mathrm{rn}}=300 \mathrm{~K}$, and an $f$-band value $10^{3} \mathrm{~Hz}$; then

$V_{\mathrm{In}} \sim 4 \times 10^{-8} \mathrm{~V}$,

which is much less than the thermal noise effective voltage $V_{\mathrm{T}}=\sqrt{2 T_{\mathrm{c}} / C} \sim 10^{-6} \mathrm{~V}$. We can conclude that, 


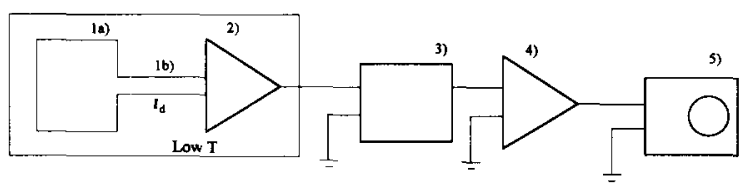

Fig. 2, RF voltage measurement scheme. 1a) A loop in the magnetic field of the superconducting RF-resonator. 1b) Waveguide (min. distance). 2) Preamplifier with narrow fband. 3) Emitter follower. 4) Resonance circuit. 5) Scope.

in practice, the minimum value of accumulated voltage is defined by the resonator thermal noise.

\section{Numerical examples}

Table 1 illustrates the values of basic parameters and requirements for different machines, assuming:

$V_{\max } / V_{\mathrm{T}}=5, \quad \xi=1, \quad \alpha=90^{\circ}, \quad d=\pi \beta c / \omega_{\mathrm{c}}$, $r_{\mathrm{c}}=20 \mathrm{~cm}$,

at a single resonator in a ring with $T_{\mathrm{c}}=1 \mathrm{~K}$. With $\mathscr{N}$ superconducting resonators, the maximum total accumulated voltage would be $\mathscr{N}$ times larger, and then the requirements for the $\Delta \nu$ value would be $\sqrt{\mathscr{N}}$ times weaker.

\section{Possible operational scheme}

An operational procedure of polarization measurement could include the following steps:

1) To swing spin coherent free oscillations, use an RF-driven voltage (perhaps a different superconducting cavity), then switch off this voltage.

2) Shunt polarimeter's superconducting cavity, in order to kill an initial RF-oscillation (excited by beam charge).

3) Turn-off the shunting resistance adiabatically.

4) Wait for spin-swing of the polarimeter superconducting cavity.

5) Measure the accumulated RF-voltage.

\section{Conclusion}

The above considerations allow us to believe that there is a physical possibility for design and use of the RF-polarimeter and hence a certain optimism regarding its technical feasibility. In the next paper, we will consider a possible development of the dynamic scheme that could bring better quality to the system of the beam spin and the RFP. To make a final determination of the RFP efficiency, the beam charge effects must also be taken into account and analyzed in detail.

\section{Acknowledgements}

I am very grateful to Profs. G. Ford, M. Tigner and J. Turneaure for helpful discussions and clarifying comments at the beginning of this investigation. I also acknowledge the help of Drs. V. Rykalin and $\mathrm{H}$. Padamsee which was quite important for understanding the feasibility of RFP. I would also like to thank Dr. A.M.T. Lin for his help in writing this paper. I am especially thankful to Prof. A.D. Krisch for stimulating interest and encouragement in performance of this work.

This work was supported by a research grant from the US Department of Energy.

\section{References}

[1] L.D. Landau and E.M. Lifshits, Course of Theoretical Physics, vol. 4 Quantum Electrodynamics, 2nd ed. (Pergamon, 1982).

[2] Ya.S. Derbenev, A.M. Kondratenko and A.N. Skrinsky, Sov. Phys. Dokl. 15 (1970) 583;

B.W. Montague, Phys. Rep. 113 (1) (1984).

[3] J.D. Jackson, Classical Electrodynamics, 2nd ed. (Wiley, 1975).

[4] H. Padamsee, private communication.

[5] L.D. Landau and E.M. Lifshits, Course of Theoretical Physics. vol. 5, Statistical Physics 3rd ed. (Pergamon, 1980).

[6] V.V. Rykalin, private communication.

Table 1

Examples

For a number $\mathscr{N}$ of resonators, the total $V$ value can be $\mathscr{N}$ times larger, the necessary accumulation time $t_{\max }$ can be $\sqrt{\mathscr{N}}$ times smaller, and the requirement for $\Delta \nu_{\min }$ is $\sqrt{\mathscr{N}}$ times weaker

\begin{tabular}{lcllll}
\hline $\begin{array}{l}\text { Machine, } \\
\text { particles }\end{array}$ & $\begin{array}{l}\text { Polarized beam } \\
\text { current [mA] }\end{array}$ & $\begin{array}{l}\text { Energy } \\
\gamma\end{array}$ & $\begin{array}{l}\dot{V} \\
{[\mathrm{~V} / \mathrm{s}]}\end{array}$ & $\begin{array}{l}t_{\text {max }}=\tau_{\mathrm{sp}}[\mathrm{s}] \\
\text { required }\end{array}$ & $\begin{array}{l}\Delta \nu_{\text {min }} \\
\text { required }\end{array}$ \\
\hline $\begin{array}{l}\text { IUCF } \\
\begin{array}{l}\text { Cooler Ring, } \\
\text { FNAL, }\end{array}\end{array}$ & 1 & 1.3 & $5 \times 10^{-6}$ & 2 & $0.5 \times 10^{-7}$ \\
$\begin{array}{l}\text { Tevatron, } \\
\text { RHIC, p }\end{array}$ & 100 & $10^{3}$ & $3 \times 10^{-4}$ & 0.03 & $10^{-4}$ \\
SSC, p & 50 & & & & $3 \times 10^{-5}$ \\
CESR, $\mathrm{e}^{ \pm}$ & 100 & 250 & $1.5 \times 10^{-4}$ & 0.06 & $1.3 \times 10^{-3}$ \\
\hline
\end{tabular}

